\title{
Crystal structure and DNA binding activity of a PadR family transcription regulator from hypervirulent Clostridium difficile R20291
}

Catherine E. Isom, ${ }^{1,}$, Smita K. Menon², Leonard M. Thomas², Ann H. West ${ }^{2}$, George B. Richter-Addo ${ }^{2}$ and Elizabeth A. Karr ${ }^{1 *}$

\begin{abstract}
Background: Clostridium difficile is a spore-forming obligate anaerobe that can remain viable for extended periods, even in the presence of antibiotics, which contributes to the persistence of this bacterium as a human pathogen during host-to-host transmission and in hospital environments. We examined the structure and function of a gene product with the locus tag CDR20291_0991 (cdPadR1) as part of our broader goal aimed at elucidating transcription regulatory mechanisms involved in virulence and antibiotic resistance of the recently emergent hypervirulent C. difficile strain R20291. cdPadR1 is genomically positioned near genes that are involved in stress response and virulence. In addition, it was previously reported that $c d P a d R 1$ and a homologue from the historical $C$. difficile strain 630 (CD630_1154) were differentially expressed when exposed to stressors, including antibiotics.

Results: The crystal structure of $c d P a d R 1$ was determined to $1.9 \AA$ resolution, which revealed that it belongs to the PadR-s2 subfamily of PadR transcriptional regulators. cdPadR1 binds its own promoter and other promoter regions from within the $C$. difficile R20291 genome. DNA binding experiments demonstrated that $c d P a d R 1$ binds a region comprised of inverted repeats and an AT-rich core with the predicted specific binding motif, GTACTAT $\left(\mathrm{N}_{2}\right)$ ATTATA(N) AGTA, within its own promoter that is also present in 200 other regions in the C. difficile R20291 genome. Mutation of the highly conserved $\mathrm{W}$ in a4 of the effector binding/oligomerization domain, which is predicted to be involved in multi-drug recognition and dimerization in other PadR-s2 proteins, resulted in alterations of $c d P a d R 1$ binding to the predicted binding motif, potentially due to loss of higher order oligomerization.
\end{abstract}

Conclusions: Our results indicate that $c d P a d R 1$ binds a region within its own promoter consisting of the binding motif GTACTAT $\left(\mathrm{N}_{2}\right)$ ATTATA(N)AGTA and seems to associate non-specifically with longer DNA fragments in vitro, which may facilitate promoter and motif searching. This suggests that $c d P a d R 1$ acts as a transcriptional auto-regulator, binding specific sites within its own promoter, and is part of a broad gene regulatory network involved, in part, with environmental stress response, antibiotic resistance and virulence.

Keywords: Clostridium difficile, Transcription regulation, Bacteria, Stress response, Structural biology, PadR

\footnotetext{
* Correspondence: lizkarr@ou.edu

${ }^{1}$ Price Family Foundation Institute of Structural Biology and Department of Microbiology and Plant Biology, University of Oklahoma, 770 Van Vleet Oval, Norman, OK 73019, USA

Full list of author information is available at the end of the article
} 


\section{Background}

Epidemiological trends indicate clinical acquisition of Clostridium difficile as the primary route of human infection by this bacterium [1]. The risk of $C$. difficile becoming a community-acquired infection is likely to increase without the development of better identification and more effective treatment [2]. The genome of $C$. difficile has been described as "highly dynamic" based on the prevalence of horizontal gene transfer [3]. The impact of a genome that readily changes in response to environmental stress could be a major indicator of $C$. difficile pathogenicity [3]. $C$. difficile produces spores that allow it to be viable for extended periods, even in the presence of antibiotics, which could explain the persistence of this human pathogen during host-to-host transmission and in the hospital environment [4]. Transcription factors orchestrate the regulation of survival, proliferation, virulence, and antibiotic resistance mechanisms of human pathogens. As part of our larger goal aimed at elucidating structure and function of transcription regulatory mechanisms involved in virulence and antibiotic resistance of human pathogens, we focused on protein targets from a hypervirulent strain of C. difficile (R20291). Herein, we present our results on a member of the PadR family of transcription regulators (product of CDR20291_0991) that we have named cdPadR1.

The first described PadR proteins are transcriptional repressors for genes encoding phenolic acid decarboxylase (padC) that de-repress padC when phenolic acids are present in toxic amounts [5]. The PadR transcription regulator from Bacillus subtilis is a prototypical PadR-family member protein that binds the padC promoter in the absence of phenolic acid in vitro; binding is lost when exposed to phenolic acids [6, 7]. Unlike the prototypical PadR, the PadR family transcription regulators AphA [8], LmrR [9], and bcPadR [10] from Vibrio cholerae, Lactococcus lactis, and Bacillus cereus, respectively, are involved in the regulation of virulence and antibiotic efflux mechanisms. The prototypical PadR and the PadR-like transcription regulator AphA are within a subfamily of PadR proteins (PadR-s1) which contain multiple $\alpha$-helices in the C-terminal domain [10]. Another, less studied subfamily of PadR family proteins (PadR-s2), contains a single $\alpha$-helix in the C-terminal effector binding/oligomerization domain [10]. The PadR-s2 proteins, which include the $b c$ PadRs [10] and LmrR [11], have been structurally characterized and are involved in multiple drug recognition. The BC4206 gene product, $b c$ PadR1, was upregulated 8.7 -fold in the presence of enterocin treatment in B. cereus ATCC14572 when compared to an untreated control [12]. This PadRlike protein binds its own promoter and that of the gene $\mathrm{BC} 4207$, which encodes a membrane protein predicted to be involved in enterocin AS-48 resistance [12]. Binding of $b c$ PadR1 to the predicted promoter region was not affected by the addition of AS-48 in vitro [10]. The PadRlike family protein of L. lactis, LmrR, binds the promoter region of an $\mathrm{ABC}$-type multidrug transporter, $\mathrm{LmrCD}$, and interacts with the compound Hoechst 33342 and the antibiotic daunomycin [9]. The crystal structure of apo-LmrR revealed a hydrophobic pore between $\alpha 4$ of the dimer mates [11]. Additional structures of LmrR bound to Hoechst 33342 and daunomycin, separately, demonstrated that this pore is integral to inhibitor interaction [11]. The conformational change instigated at $\alpha 4$ is predicted to interfere with DNA binding due to an increase in distance between $\alpha 3$ of the dimer mates [13]. This hydrophobic pore is not present in $b c$ PadR structures determined to date.

The genome of hypervirulent $C$. difficile R20291 contains the protein coding sequence for three PadR-like family proteins (cdPadR1, CDR20291_1187, CDR20291_3068). The function of $c d$ PadR1 is of interest due, in part, to its similarity to previously described $b c$ PadRs and LmrR and the response of these transcription regulators to multiple inhibitors. Importantly, differential expression studies have linked $c d$ PadR1 and a homologue from historical $C$. difficile strain 630 (CD630_1154) to regulatory networks that allow $C$. difficile to efficiently respond to environmental changes and, thus, survive within a host. This response is not necessarily due to direct interaction with stressors, but may be part of an overall regulatory cascade. Germination of $C$. difficile strain 630 endospores lead to the differential expression of 92 different transcriptional regulators, $\sim 74 \%$ of which were up-regulated as detected by microarray and validated by qRT-PCR [14]. Included in this list of differentially expressed transcription regulators is the $c d$ PadR1 homologue CD630_1154, which was 2.3-fold up-regulated during germination [14]. This suggests that the expression of one or more of these proteins required to bring an endospore out of dormancy may be regulated by CD630_1154. Another study linked the differential expression of this $c d$ PadR1 homologue to acid and alkali shock, oxygen exposure, and subinhibitory concentrations of metronidazole (Mtz) as detected by microarray analyses in C. difficile strain 630 [15].

Herein, we investigated the PadR-s2 protein from $C$. difficile strain R20291, cdPadR1. In this paper, we report the crystallization and X-ray crystal structure of $c d$ PadR1 at $1.9 \AA$ resolution. We also demonstrate $c d$ PadR1 binding to its own gene promoter in a manner conducive to autoregulation. Additionally, we show that $c d$ PadR1 binds the promoters of three additional regulatory signaling proteins and that a $c d \operatorname{PadR} 1$ binding motif is present upstream of 100 genes in C. difficile R20291.

\section{Methods}

Protein expression and purification

Residues 1-109 of $c d$ PadR1 (locus tag CDR20291_0991) were amplified from gDNA using forward primer 
Pr3 -EAK (5'- TTCAGGGATCCATGCAGTTAAA TAAAGAAGTGTTAAAAGG-3') and reverse primer Pr4-EAK (5'-TTAAGCTGCAGTTAATCCACCTCTCC CAAAAATTG-3') primers, each of which contained a 5 nucleotide overhang followed by restriction digestion sites for BamHI (forward) or PstI (reverse) for digestion and ligation into the expression vector. $c d \mathrm{PadR} 1$ was expressed in Escherichia coli Rosetta $^{\mathrm{Tm}}$ using the $\mathrm{pQE80L}$ (Qiagen) vector system modified to encode a Strep II $^{\mathrm{m}}$-tag on the $\mathrm{N}$-terminus [16]. $c d$ PadR1 was isolated by batch purification over Streptactin SuperFlow Plus resin (Qiagen). All buffers were prepared according to the manufacturers' guidelines. Cell lysis, column equilibration, and wash buffer contained $50 \mathrm{mM} \mathrm{NaH} \mathrm{PO}_{4}$ and $300 \mathrm{mM} \mathrm{NaCl}$ (pH 8.0 using $\mathrm{NaOH}$ ). Elution buffer contained $50 \mathrm{mM}$ $\mathrm{NaH}_{2} \mathrm{PO}_{4}, 300 \mathrm{mM} \mathrm{NaCl}$, and $2.5 \mathrm{mM}$ d-desthiobiotin $(\mathrm{pH} 8.0$ using $\mathrm{NaOH})$. Subsequent purification of the $c d$ PadR1 dimer was accomplished by size exclusion chromatography in buffer containing $20 \mathrm{mM}$ Tris ( $\mathrm{pH} 8.0$ with $\mathrm{NaOH}$ ) and $150 \mathrm{mM} \mathrm{NaCl}$, using a Superdex 200 Increase 10/300 GL column connected to an ÄKTA Pure 25 (GE Healthcare). Fractions corresponding to a dimer were concentrated using Amicon ${ }^{\bullet}$ concentration units (Millipore) primed with glycerol and buffer exchanged into $10 \mathrm{mM}$ Tris ( $\mathrm{pH} \mathrm{8.0)}$ and $100 \mathrm{mM} \mathrm{KCl}$. The molecular weight (MW) was determined by coupling SEC with multi-angle light scattering (MALS) and outputs were analyzed by the ASTRA software (Wyatt Technology).

\section{Crystallization of $c d$ PadR1}

Crystals were initially obtained by vapor diffusion using a MCSG Crystallization Suite (Microlytic) (3 M NaCl and 0.1 M HEPES pH 7.5) with a final protein concentration of $1.5 \mathrm{mg} \mathrm{mL}^{-1}$. Crystal growth was optimized at room temperature by hanging drop vapor diffusion with the drops containing $3 \mu \mathrm{L}$ protein solution $\left(4 \mathrm{mg} \mathrm{mL}^{-}\right.$ ${ }^{1} c d$ PadR1 in $100 \mathrm{mM} \mathrm{KCl}, 10 \mathrm{mM}$ Tris $\left.\mathrm{pH} 8.0\right)$ and 1 $\mu \mathrm{L}$ reservoir solution $(3.1 \mathrm{M} \mathrm{NaCl}, 100 \mathrm{mM}$ HEPES [pH 7.5]). Crystals were transferred into drops containing an equal volume of $2 \mathrm{X}$ reservoir solution and $40 \%$ glycerol for cryoprotection. Crystals in cryosolution were incubated over original well solution for $5 \mathrm{~min}$ before freezing in a liquid nitrogen gas stream for cryogenic data collection.

\section{Data collection and structure determination}

X-ray diffraction data were collected using a MARmosaic325 CCD detector at the Stanford Synchrotron Radiation Lightsource (SLAC National Accelerator Laboratory) on beam-line BL14-1. The data were processed with XDS and XSCALE [17]. The XDS output files were converted to .mtz format using CCP4 [18]. The structure of Clostridium thermocellum PadR-like family protein (CtPadR, PDB ID 1XMA) was used as the starting model for molecular replacement using Phaser-MR [19]. The individual coordinates of the preliminary model were generated in AUTOBUILD [20], were refined and rebuilt using the model in COOT [21] and any positions with strong densities outside of the model were accounted for. Structure alignments were performed in COOT and all structure/alignment figures prepared using PyMOL [22]. Residues 1-9, 41, and 107-109 were not modeled due to the absence of electron density. Coordinates have been deposited with the Protein Data Bank (www.rcsb.org) with PDB ID 5DYM. Data collection and refinement statistics are shown in Table 1.

\section{Construction of $c d P a d R 1^{\text {W94A }}$}

The tryptophan 94 (W94) codon (TGG) of $c d$ PadR1 was converted to alanine (GCG) by overlapping PCR [23]. The sequence of forward and reverse primers used to generate the alanine codon substitutions in $c d$ PadR1 were 5 '-GAAACAAGAAGCGAGATTTATTAAAAAG-3' and 5'-CTTTTTAATAAATCTCGCTTCTTGTTTC-3', respectively. The resulting plasmid was confirmed by sequencing, and the resulting protein variant was overexpressed and purified in the same manner as performed for the native $c d$ PadR1.

\section{Electrophoretic Mobility Shift Assay (EMSA)}

Double stranded DNA fragments for EMSA were generated by suspending custom complementary ssDNA (LifeTechnologies) in annealing buffer $(10 \mathrm{mM}$ Tris $[\mathrm{pH}$ 8.0] and $50 \mathrm{mM} \mathrm{NaCl}$ ) and heating to $95{ }^{\circ} \mathrm{C}$ for $5 \mathrm{~min}$ followed by slowly cooling to room temperature. DNA was quantified with the Quant-IT ${ }^{\mathrm{Tm}}$ Broad Range DNA assay and a $\mathrm{Qubit}^{\circ}$ fluorimeter (Invitrogen). Template dilutions for EMSA stock solutions were dependent on the size of the DNA fragment and ranged from $0.5 \mu \mathrm{M}$ (100 bp fragment) to $2.5 \mu \mathrm{M}$ (20 bp fragment). Binding reactions were performed at room temperature. Each reaction mixture contained $20 \mathrm{mM}$ Tris $\mathrm{pH} 8.0,120 \mathrm{mM}$ $\mathrm{KCl}, 12.5 \%$ glycerol, $10 \mathrm{mM} \mathrm{MgCl}$, $5 \mathrm{mM} \mathrm{DTT}$, and $125 \mu \mathrm{g} \mathrm{mL}{ }^{-1}$ heparin. Heparin concentration was increased to $400 \mu \mathrm{g} \mathrm{ml}^{-1}$ for competition studies. A 1:10 dilution of DNA stock was added to all reactions and a $c d$ PadR1 concentration 2.5-40-fold greater than that of final DNA concentration was added to start the binding reaction. A protein-free control was also included. EMSAs were performed in $8 \%$ polyacrylamide gels and TB running buffer $(89 \mathrm{mM}$ Tris base and $89 \mathrm{mM}$ boric acid) at $200 \mathrm{~V}$ and $20-100 \mathrm{~mA}$ with run time ranging from $20 \mathrm{~min}$ (20 bp fragments) to $30 \mathrm{~min}$ (100 bp fragments). Gels were stained with $\mathrm{SYBR}^{\circ}$ Gold Stain (Invitrogen). Image coloration was inverted for ease of viewing. A list of oligonucleotides examined, including location on the genome, sequences, and GC content (\%) can be found in Additional file 6: Table S1. 
Table 1 Data collection and refinement statistics

\begin{tabular}{|c|c|}
\hline \multicolumn{2}{|c|}{ Data collection statistics (SSRL BL14-1) } \\
\hline Wavelength (Å) & 1.2 \\
\hline Space group & $P 4,2,2$ \\
\hline \multicolumn{2}{|l|}{ Unit cell dimensions } \\
\hline$a, b, c(\AA)$ & $92.4,92.4,40.4$ \\
\hline$a, \beta, y\left(^{\circ}\right)$ & 90 \\
\hline Resolution $(\AA ̊)$ & $34.4-1.9(2.0-1.9)^{\mathrm{a}}$ \\
\hline$R_{\text {merge }}^{b}$ & $0.019(0.38)$ \\
\hline $\mid / \sigma[l]$ & $20.3(2.2)$ \\
\hline Completeness (\%) & $99(95)$ \\
\hline Redundancy & $2.0(2.0)$ \\
\hline Wilson $B(\AA)$ & 33.1 \\
\hline \multicolumn{2}{|l|}{ Refinement statistics } \\
\hline Resolution range $(\AA)$ & $34.4-1.9(2.0-1.9)$ \\
\hline \multicolumn{2}{|l|}{ No. of reflections } \\
\hline Total & $28484(2641)$ \\
\hline Unique & $14302(1332)$ \\
\hline$R_{\text {work }}^{C}(\%)$ & $0.21(0.29)$ \\
\hline$R_{\text {free }}^{\mathrm{d}}(\%)$ & $0.23(0.34)$ \\
\hline \multicolumn{2}{|l|}{ No. of atoms } \\
\hline Total (non-H) & 842 \\
\hline Water molecules & 34 \\
\hline \multicolumn{2}{|l|}{ Mean overall B factors } \\
\hline Protein & 49.3 \\
\hline Solvent & 43.3 \\
\hline \multicolumn{2}{|l|}{ RMSDs } \\
\hline Bond length $(\AA)$ & 0.008 \\
\hline Bond angles $\left(^{\circ}\right)$ & 1.03 \\
\hline \multicolumn{2}{|l|}{ Ramachandran plot } \\
\hline$\%$ most favored residues & 96 \\
\hline$\%$ outliers & 0 \\
\hline PDB code & 5DYM \\
\hline
\end{tabular}

${ }^{a}$ Statistics for data in the highest resolution shell are given in parentheses

${ }^{\mathrm{b}} R_{\text {merge }}=\left(\Sigma I_{\text {obs }}-l_{\text {avg }}\right) / \Sigma l_{\text {avg }}$

${ }^{c} R_{\text {work }}=\left(\Sigma F_{\text {obs }}-F_{\text {calc }}\right) / \Sigma F_{\text {obs }}$

${ }^{\mathrm{d}} R_{\text {free }}$ was calculated using the test set obtained by randomly selecting $10 \%$ of the data

\section{Motif Analysis}

GLAM2 was utilized to find a representative $c d$ PadR1 motif [24]. The sequence surrounding Boxes 1 \& $2\left(5^{\prime}\right.$-GTACTA TACATTATAGAGTAGTAG-3') and Boxes 3 \& 4 (5'AGAGTACTATGTATTATTATAGTAAAT-3') were used as input sequences for the GLAM2 analysis. The GLAM2 search was done using the default parameters and allowed the motif sites to be on either the plus or minus strand. The direct GLAM2 output was used as the input for GLAM2 Scan using the C. difficile R20291 genome. Motifs were allowable on either the minus or plus strand of the genome and 200 alignments were allowed. The identified motifs were then mapped onto the $C$. difficile R20291 genome sequence in Geneious v8 [25]. The motifs were then manually curated to determine whether they were located within an open reading frame, an intergenic promoter region or between convergent genes.

\section{Results and discussion}

\section{Crystal structure of recombinant $c d P a d R 1$}

cdPadR1 shares $100 \%$ amino acid sequence identity with the PadR-like transcription regulator, CD630_1154, in the historical C. difficile strain 630 (Fig. 1), both of which were differentially expressed under conditions of environmental stress [15]. cdPadR1 crystallized in space group $\mathrm{P}_{1} 2_{1} 2$ and, following X-ray data collection, the structure was solved by molecular replacement using the PadR family protein from $C$. thermocellum (CtPadR) as a search model (PDB ID 1XMA). CtPadR and $c d$ PadR1 share $42 \%$ amino acid sequence identity (Fig. 1) and, based on 3D prediction programs [26, 27], were expected to have high structural similarity $(\mathrm{RMSD}=1.7 \AA$ ). The model was refined to a final crystallographic R-factor of $21.0 \%\left(R_{\text {free }}=23.0 \%\right)$ (Table 1$)$.

One molecule of $c d \operatorname{PadR} 1$ was present in the asymmetric unit and consists of an N-terminal winged helixturn-helix (wHTH) domain (residues 6-80) and a single $\alpha$-helical C-terminal domain (residues 81-106) (Fig. 2a). This small C-terminal domain places $c d$ PadR1 in the PadR-s2 subfamily of PadR transcriptional regulators described previously [10]. cdPadR1 forms a dimer with a 2-fold crystallographic axis of symmetry (Fig. 2b), similar to the $b c$ PadRs (PDB IDs 4ESB and 4ESF) and LmrR (PDB ID 3F8B), both of which are PadR-s2 family proteins. The dimeric state of $c d \operatorname{PadR} 1$ is retained in solution as determined by size exclusion chromatography (Additional file 1: Figure S1). The recognition helices $\left(\alpha 3 / \alpha 3^{\prime}\right)$ are positioned $\sim 34 \AA$ apart (Fig. 2b) consistent with symmetrical binding to two "half-sites" approximately $10 \mathrm{bp}$ in length [28]. Dimerization of $c d \operatorname{PadR} 1$ buries approximately $1100 \AA^{2}$ solvent-accessible surface area $(16 \%)$ of the approximately $7000 \AA^{2}$ total solventaccessible area per subunit [29]. Residues on helices $\alpha 1$, $\alpha 2$, and $\alpha 4$ that interact to form the $c d \operatorname{PadR} 1$ dimer interface are conserved across structural homologues (Fig. 1). The RMSD values for the $c d$ PadR1 structural homologues $b c$ PadR1, $b c$ PadR2, apo-LmrR, LmrRH33342, and LmrR-daunomycin are $1.6 \AA, 1.6 \AA, 2.1 \AA$, $2.9 \AA$, and $3.3 \AA$, respectively [27].

The primary helices involved in dimerization are $\alpha 1$ and $\alpha 4$. The amino acid sequence pairwise identities between $\alpha 1$ of $c d$ PadR 1 and $b c$ PadR1, $b c$ PadR2, and LmrR are $26 \%$, $35 \%$, and $21 \%$, respectively. The amino acid sequence pairwise identity between $\alpha 4$ of $c d \operatorname{PadR} 1$ and $b c$ PadRs (22.2 and $33.3 \%$ for $b c$ PadR1 and $b c$ PadR2, 


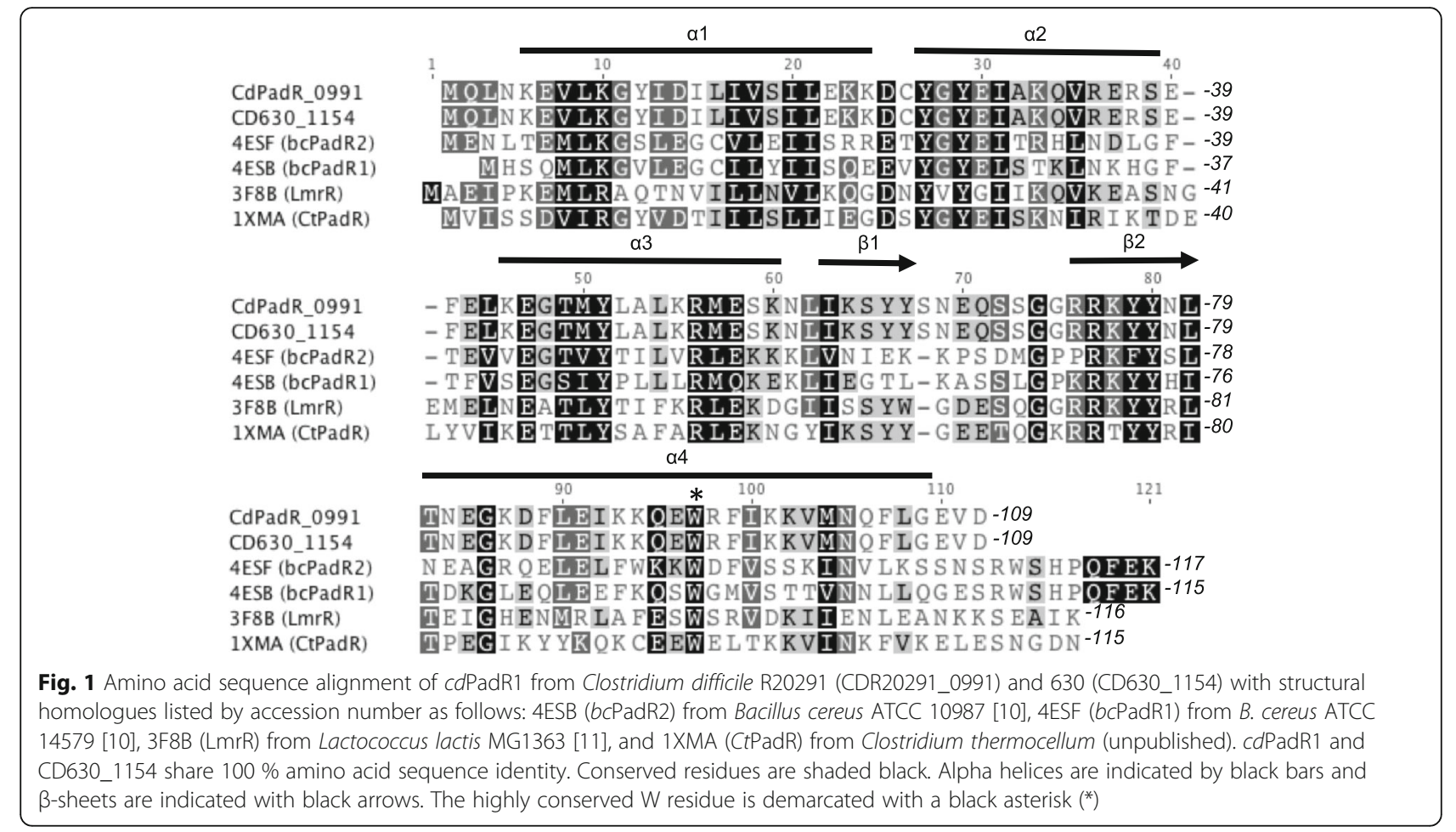

respectively) is higher than the identity between $\alpha 4$ of $c d$ PadR1 and LmrR (15\%). Helix $\alpha 4$ and $\alpha 4^{\prime}$ of $c d$ PadR1 bend toward each other (Fig. 2b) and interact via a coiled-coil, whereas $\alpha 4$ and $\alpha 4^{\prime}$ of LmrR do not display a significant bending towards each other at the $C$ terminus (Fig. 3a, red). In addition, LmrR contains fewer residues involved in dimerization at the $\mathrm{C}$-terminus of the helix than $c d$ PadR1 and $b c$ PadRs. $c d$ PadR1, like $b c$ PadRs and $c t$ PadR, has a closed dimeric interface, unlike the hydrophobic pore wherein aromatic druginteraction occurs in LmrR (Fig. 3b). The known structural homologues of $c d$ PadR1 contain a conserved W located within residues 91-96 in the $\alpha 4$ helix region that is predicted to be involved in both dimerization and drug binding $[10,11]$. The distance between the conserved W residues in the $\alpha 4$ helix dimer mates for $c d$ PadR1, bcPadR1 (4ESB), apo-LmrR (3F8B), LmrRH33342 (3F8C), and LmrR-daunomycin (3F8F) was measured using Chimera [30]. Distances were determined from the centroids of the phenol rings (P-P), indole rings (I-I), and indole-to-phenol (I-P) of the conserved $\alpha 4 \mathrm{~W}$ residues. The P-P, I-I, and I-P distances between cdPadR1 W94 and W94' are 5.4 $\AA, 9.2 \AA$, and 7.4 $\AA$, respectively. These distances are similar to those of $b c$ PadR1 (P-P $=5.6 \AA$, I-I = 9.1 $\mathrm{A}$, and $\mathrm{I}-\mathrm{P}=7.4 \AA$ ). The P-P distance is $\sim 2 \AA$ greater for the apo-LmrR (P-P $=6.9$ $\AA$ ), LmrR-H33342 (P-P = $7.2 \AA$ ), and LmrR-daunomycin (P-P $=7.4 \AA$ ) structures when compared to the distance between phenol centroids in $c d$ PadR1. The increased distance between $\alpha 4$ and $\alpha 4^{\prime}$ of LmrR allows for aromatic inhibitor interaction via $\pi$-stacking between the W96 and W96' residues [11]. The lack of a drug-binding pocket in $c d$ PadR1 suggests that any differential expression of the CD630 homologue (CD630_1154) during Mtz exposure would, most likely, be due to a regulatory cascade effect rather than direct interaction of $c d$ PadR1 with Mtz. It was suggested that changes in the orientation of $\alpha 4$ and $\alpha 4$ 'in a drug-bound state effects the position of the DNA recognition helices, rotating them away from each other [11]. This, presumably, would cause a change in DNA-binding. Previous work revealed that LmrR binds two sites within the $\operatorname{lm} r C D$ promoter, one region containing the predicted -10 and -35 sites and the other containing the inverted repeats ATGT/ ACAT separated by 10 nucleotides and that this is consistent with a "conserved" binding motif among other PadR-like regulators with an eight nucleotide linker between the inverted repeats ATGT/ACAT [9, 31]. The recognition helices $(\alpha 3 / \alpha 3$ ) are positioned $\sim 34 \AA$ apart in $c d$ PadR1 (Fig. 2b), which is consistent with symmetrical binding to two "half-sites" comprised of inverted repeats $\sim 10$ bp apart; it is important to note that this distance does not account for DNA secondary structure. DNA binding behavior was explored for $c d$ PadR1 to determine if it functions similarly to previously studied PadR family transcription regulators and to begin elucidating the regulatory networks of $c d \operatorname{PadR} 1$ in hypervirulent $C$. difficile in vitro. 


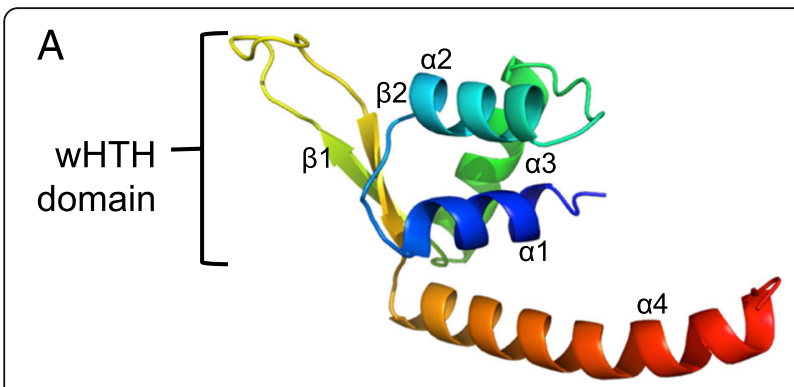

B

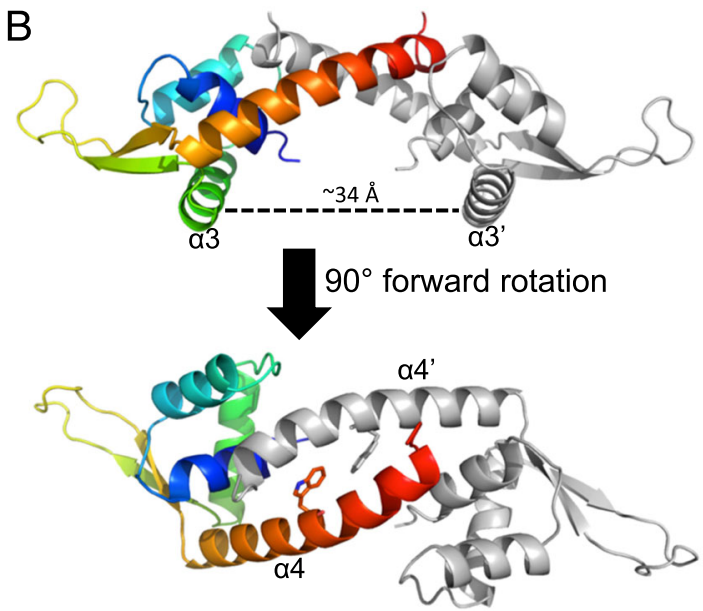

Fig. 2 Overall structure of $c d P a d R 1$. a Ribbon representation of $c d P a d R 1$ monomer with a rainbow color gradient from the N-terminus (blue) to the C-terminus (red). Alpha helices and $\beta$-sheets are labeled numerically. The winged helix-turn-helix (wHTH) DNA binding domain is indicated. b The $c d P a d R 1$ structure is shown perpendicular to the two-fold axis of symmetry with the DNA recognition helices indicated (a3/a3'). The dimer mate is shown in gray. Distance between a3/a3' was estimated in PyMOL [22]. Another view is shown after a $90^{\circ}$ forward rotation which results in a view along the two-fold axis facing a4/a4' with the conserved $W$ residue (sticks)

\section{cdPadR1 binding to its own promoter}

A 100 bp region upstream of cdpadR1 ( $\left.\mathrm{P}_{\text {cdpadR1 }} / \operatorname{Pr} 27\right)$ was used in EMSA assays to determine if $c d$ PadR1 binds its own promoter (Fig. 4a). The presence of five bands with differing mobility indicated that protein-DNA complexes of varying stoichiometry were produced. This may be the result of multiple binding sites and/or higher order oligomerization upon DNA binding (Fig. 4b). Increasing the concentration of $c d \mathrm{PadR} 1$ in the reaction resulted in a variation of the migration pattern until an observed saturation point at the slowest mobility compared to other bands was achieved (40-fold $c d$ PadR over DNA or $4 \mu \mathrm{M} c d$ PadR1, Fig. $4 \mathrm{~b}$, far right). $c d$ PadR1 binding to $\mathrm{P}_{\text {cdpadR1 }}$ (Pr27) is consistent with autoregulation of its own expression.

To further define the binding sites for $c d$ PadR1 binding to $\mathrm{P}_{c d p a d R 1}$, Palinsight was used to identify inverted repeats within Pr27 characteristic of those bound by transcriptional regulators containing a HTH motif [32-34]. Two

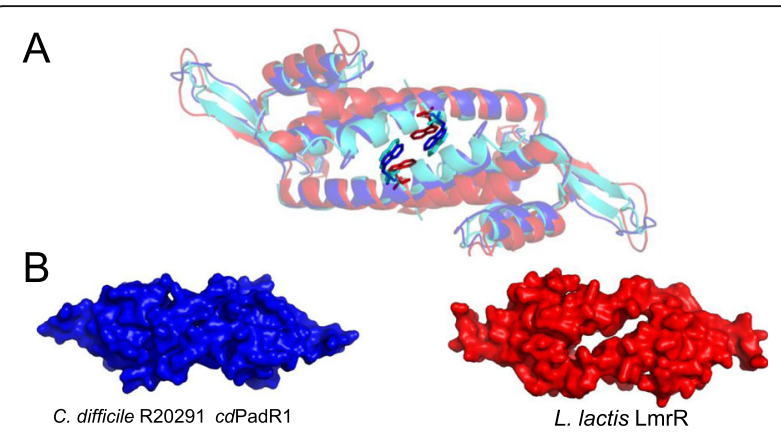

Fig. 3 Differences between $c d P a d R 1, b c P a d R 1$ (PDB ID 4ESB), and apo-LmrR (PDB ID 3F8B). a Superposition of cdPadR1 (blue), bcPadR1 (cyan), and LmrR (red) in cartoon representation with the conserved W indicated (sticks). b Surface representations of $c d P a d R 1$ and apo-LmrR along the two-fold axis facing a4/a4' (orientation same as Fig. 3c), which highlights the hydrophobic pore in LmrR and the closed dimeric interface of cdPadR1

sets of inverted repeats (Box $1 / 2$ and 3/4) were identified with a TACT $\left(\mathrm{N}_{11-12}\right)$ AGTA sequence motif (Fig. 4a). A series of smaller dsDNA fragments within the $100 \mathrm{bp}$ $\mathrm{P}_{\text {cdpadR1 } 1}$ were designed to test the role of these inverted repeats in $c d$ PadR1 binding to $\mathrm{P}_{c d p a d R 1}$ (Fig. 4a). A 64 bp fragment containing both sets of inverted repeats (Pr32) showed four shifts of varying stoichiometry similar to that seen for Pr27 (Fig. 4c). However, full saturation, as seen for Pr27, was not achieved suggesting that additional space on the DNA for higher order oligomerization is needed to see complete shifting to one higher molecular weight complex. When $c d$ PadR1 bound a 61 bp fragment that contained only one set of inverted repeats (Pr31) three shifted complexes were observed (Fig. 4d). This is consistent with the loss of a full binding site and additional space on the DNA for higher order oligomerization as noted for Pr31.

We further narrowed $c d$ PadR1 binding to two small regions of $\mathrm{P}_{\text {cdpadR1 }}$ (Pr68 and Pr122) each containing one set of inverted repeats TACT( $\left.\mathrm{N}_{11-12}\right)$ AGTA (Fig. 4a). $c d$ PadR1 bound the 21 (Pr68) and $30 \mathrm{bp}(\operatorname{Pr} 122)$ regions of $\mathrm{P}_{c \text { dpadR1 }}$ with a single stoichiometry as visualized using EMSA (Fig. 4e and f, respectively). Additionally, a variety of dsDNA fragments representing various subregions of the original $100 \mathrm{bp} \mathrm{P}_{\text {cdpadR1 }}$ (Pr27) were examined and, unless the fragment contained the predicted inverted repeats TACT $\left(\mathrm{N}_{11-12}\right)$ AGTA, no binding was observed (Fig. 4g). It was noted that the $\mathrm{N}_{11-12}$ spacer region within the inverted repeats was AT rich. To determine whether the AT richness contributes to localized bending of the DNA that facilitates binding we replaced the TTATA in Pr68 with a GCCTG sequence (Pr101). Indeed, significant binding of $c d \operatorname{PadR} 1$ to Pr101 was not observed (Additional file 2: Figure S2) suggesting that the AT-rich spacer is important for binding. It should be noted that a fragment containing the AT rich portion 

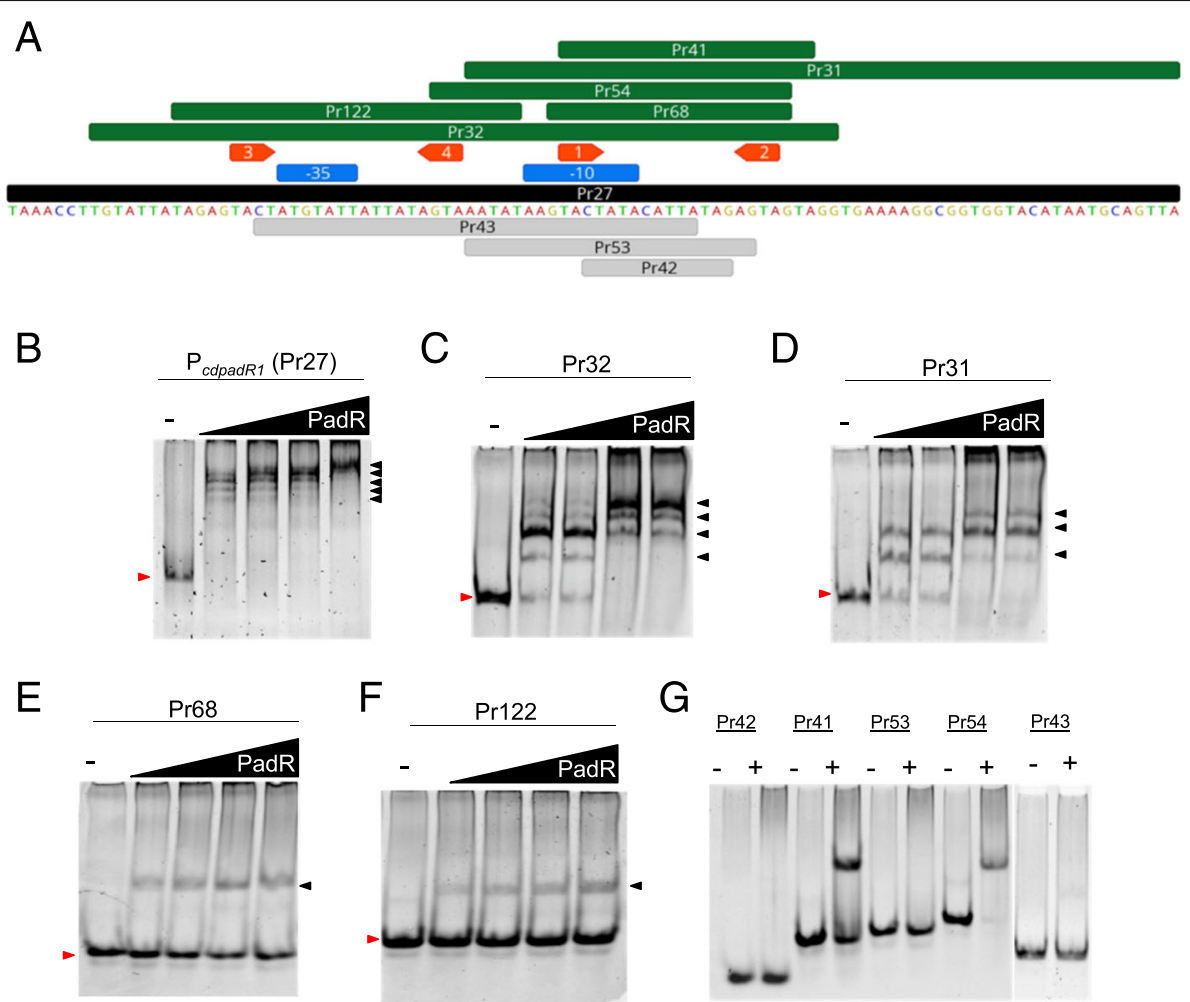

Fig. 4 EMSAs of $c d P a d R 1$ binding the $c d p a d R 1$ promoter $\left(P_{c d p a d R 1}\right)$ and smaller regions containing predicted binding boxes. a $P_{c d p a d R 1}$ fragments that were bound by $c d P a d R 1$ are illustrated in green above and those that were not bound are illustrated in gray below the $P_{c d p a d R 1}$ sequence. The predicted -10 and -35 sites are indicated in blue boxes above the sequence. $\mathbf{b}$ Final $P_{\text {cdpadR }}$ (Pr27, 100 bp) concentration in the reaction was $0.05 \mu \mathrm{M}$. cdPadR1 was 5, 10, 20, and 40-fold excess over DNA. c-d Final Pr32 (64 bp) and Pr31 (61 bp) concentration in the reactions were $0.1 \mu \mathrm{M}$.

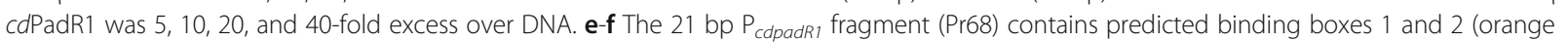
arrows) separated by $11 \mathrm{bp}$ and contains a 1 bp overhang on both the $5^{\prime}$ and $3^{\prime}$ ends. The 30 bp P cdpadR1 fragment (Pr122) contains predicted binding boxes 3 and 4 (orange arrows) and contains 5 bp overhangs on both the $5^{\prime}$ and $3^{\prime}$ ends. Final Pr68 (21 bp) and Pr122 (30 bp) concentration in the reactions were $0.25 \mu \mathrm{M}$. cdPadR1 was 2, 4, 6, and 8-fold excess over DNA. $\mathbf{g}$ Final dsDNA concentration varied depending on the size of the fragment; the + lane contains 10-fold excess cdPadR1 over DNA. For EMSA gels B-F the minus (-) lane contains DNA and no cdPadR1. Shifted DNA-protein complexes are annotated with a black arrow and unbound DNA migration is marked with a red arrow

but lacking the intact TACT/AGTA (Pr42) was not bound by $c d$ PadR1 (Fig. 4a and g). This indicates that the AT rich sequence is not the direct binding site for $c d$ PadR1. Additionally, varying the length of the spacer between the TACT/AGTA inverted repeat in Pr68 did not interfere with binding (Additional file 2: Figure S2) suggesting that flexibility of the DNA region between the inverted repeat rather than the length is more important for $c d$ PadR1 binding.

To summarize, $c d$ PadR1 binding to $\mathrm{P}_{c d p a d R 1}$ is dependent upon a TACT/AGTA inverted repeat sequence. Two such sequences are present in the $100 \mathrm{bp} \mathrm{P}_{c d p a d R 1^{-}}$ investigated in this study. These two inverted repeats are responsible for two sequence-specific interactions between $c d$ PadR1 and its promoter that can account for two shifted complexes. Additional shifted complexes may be the result of higher order oligomerization of $c d$ PadR1 once bound to DNA or a decrease in the constraints on sequence specificity. Although constraints on the spacing between the TACT/AGTA inverted repeats do not appear to be tight, there does appear to be a requirement for AT richness within the spacer. The placement of the inverted repeat within $\mathrm{P}_{\text {cdpadR1 }}$ is consistent with auto regulation. $c d$ PadR1 and the $c d$ PadR1 homologue CD630_1154 both contain TACT/AGTA with an 11 nucleotide spacer 25 bp upstream of the open reading frame (ORF). Additionally, both promoter regions contain TACT $\left(\mathrm{N}_{12}\right)$ AGTA $52 \mathrm{bp}$ upstream of their respective ORFs and overlapping the predicted $-35 /-10$ promoter region, which suggests a similar binding function for each of these genes to their respective promoters.

\section{$c d \mathrm{PadR} 1$ binds other gene promoters with the $c d \mathrm{PadR} 1$ motif}

The dsDNA fragments containing TACT $\left(\mathrm{N}_{11-12}\right)$ AGTA from $P$ cdPadR1 were analyzed for conserved binding motif using GLAM2 [24]. GLAM2 was advantageous over MEME because it allows for spacing/gaps in motif 
prediction since spacing between the inverted repeats was not critical for binding. The best motif was $21 \mathrm{bp}$ in length with the sequence GTACTAT $\left(\mathrm{N}_{2}\right) \operatorname{ATTATA}(\mathrm{N})$ AGTA and was designated $c d$ PadR1 motif (Fig. 5a). GLAM2Scan results indicated the presence of 200 potential motif matches in the $C$. difficile strain R20291 genome with scores ranging from 13.6-18.7, not including the $\mathrm{P}_{\text {cdpadR } 1}$ sequences used for analysis (Additional file 3: Table S2). Approximately half of these motifs are either situated between two convergent genes or are located within open reading frames (ORFs). Of those that are located upstream of genes, approximately $6 \%$ are upstream of other transcription regulators and other regulatory proteins, such as two-component response regulators, while another $\sim 7 \%$ are upstream of genes involved in transport/efflux and sporulation. The genes predicted to be involved in transport/efflux are the ABC transporter ATPbinding proteins CDR20291_0159, _0296, _0551, _0553, and 3203 (Additional file 4: Table S3). Two genes predicted to be involved in sporulation also contain the $c d$ PadR1 binding motif upstream of the transcription start site, a spore maturation protein (CDR20291_3377) and a spore coat assembly protein (CDR20291_0316) (Additional file 4: Table S3). Over $50 \%$ of the predicted binding motifs were indicated to be either upstream genes of "hypothetical proteins", within open reading frame, or between convergent genes. Exemplar promoters from this list were selected for analysis using EMSA to determine binding of $c d$ PadR1 to these promoter fragments in vitro (Fig. $5 \mathrm{~b}$ and c). A $30 \mathrm{bp}$ and $100 \mathrm{bp}$ dsDNA fragment was selected for each promoter region and contained at least one predicted $c d$ PadR1 motif (Fig. 5b and c). Pr132 and Pr133 contain the $c d$ PadR1 motif located 45 base pairs upstream of CDR20291_2322 (IclR family transcription regulator CDS) $\left(c d \mathrm{P}_{2322}\right)$. Pr135 and Pr136 contain the $c d$ PadR1 motif located 116 base pairs upstream of CDR20291_1882 (two-component system response regulator CDS) $\left(c d \mathrm{P}_{1882}\right)$. Pr137 and Pr138 contain the $c d \operatorname{PadR} 1$ motif located 25 base pairs upstream of CDR20291_1590 (ArsR family transcriptional regulator CDS) $\left(c d \mathrm{P}_{1590}\right)$. $c d \mathrm{PadR} 1$ bound all of the selected promoters in vitro (Fig. $5 \mathrm{c}$ ). The $30 \mathrm{bp}$ promoters (Pr132, Pr135, Pr137) yielded two discrete bands. However, this phenomenon has also been observed on occasion for the short dsDNA fragment containing one set of
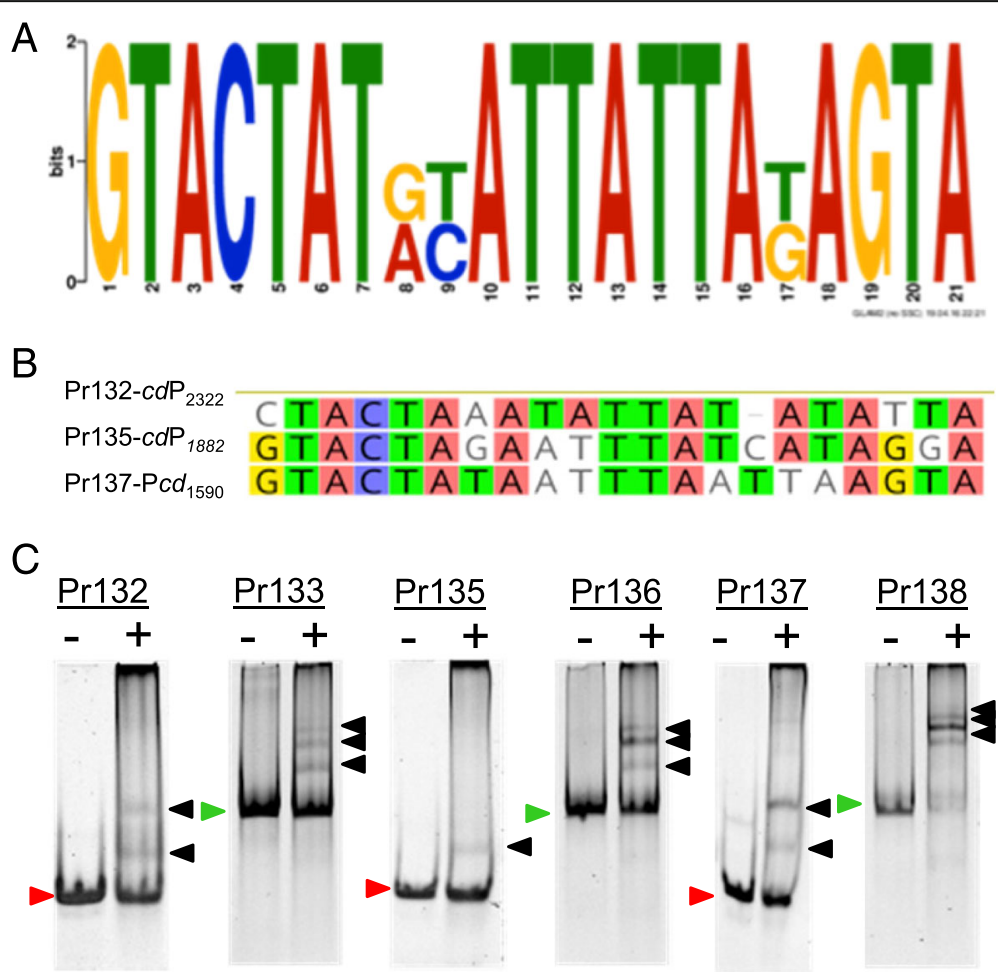

Fig. 5 Example analysis of predicted $c d P a d R 1$ binding motif ( $c d P a d R 1$ motif) as verified by EMSA. a Motif identified by GLAM2 analysis [24] of small dsDNA fragments from within the cdPadR1 promoter (Pr68 and PR122). b cdPadR1 motifs present in three exemplar promoters compared to the $c d P a d R 1$ motif. Nucleotides that are in agreement with the reference $c d P a d R 1$ motif are highlighted. Pr132 and Pr133 are upstream of CDR20291_2322 (ICIR family transcription regulator CDS); Pr135 and Pr136 are upstream of CDR20291_1882 (two-component system response regulator CDS); Pr137 and Pr138 are upstream of CDR20291_1590 (ArsR family transcriptional regulator CDS). b EMSAs of cdPadR1 binding 30 bp (green arrows) and $100 \mathrm{bp}$ (red arrows) promoter regions that contain cdPadR1 motif. DNA only (-) reactions contained $100 \mathrm{nM}$ either 30 or 100 bp dsDNA from promoters listed above. Reactions containing dsDNA and $4 \mu \mathrm{M} c d P a d R 1$ are noted with a plus (+) sign. Final DNA concentration in both reactions was $100 \mathrm{nM}$. Bound complexes are indicated with black arrows 
inverted repeats from $\mathrm{P}_{c d p a d R 1}$ (Pr68, Additional file 5: Figure S3) and is not likely to represent multiple binding events to a small dsDNA fragment [35]. This binding pattern may be attributable to the presence of small amounts of ssDNA, portions of the dsDNA with secondary structure, or conformational changes in the DNA upon binding in a small subset of the complexes which are more pronounced in shorter dsDNA fragments [35].

Gene regulatory networks play an integral role in the physiology of microorganisms and their response to ever changing environments [36, 37]. The binding of $c d \operatorname{PadR} 1$ to the promoters of genes encoding transcription regulators and a DNA-binding response regulator, part of a twocomponent signal transduction system, suggests it may play a role in a gene regulatory network in $C$. difficile. The $c d$ PadR1 motif overlaps the predicted -10 region of $c d \mathrm{P}_{1590}$ and $c d \mathrm{P}_{2322}$ [38]. This positioning of a regulatory binding site overlapping the -10 region is consistent with repression via abrogation of the Sigma factor. In $c d \mathrm{P}_{1882}$, the $c d \operatorname{PadR} 1$ motif is located approximately $30 \mathrm{bp}$ upstream of the predicted -35 region [38]. Positioning of a regulatory binding site upstream of the $-35 /-10$ core promoter elements is typically consistent with a role in activation of the promoter [39]. While additional studies are necessary to determine the biological role of $c d \operatorname{PadR} 1$ in activation or repression of these promoters, it is notable that $c d \mathrm{PadR} 1$ is able to bind these promoters and likely participates in a regulatory cascade in response to undetermined stimuli.

\section{cdPadR1 binds other promoter regions}

Additional promoters from the cdpadR1 genomic neighborhood were chosen to test for $c d$ PadR1 binding based on gene expression studies. A promoter for a nitric oxide reductase (norV, CDR20291_0994) and a Spo0Bassociated GTP-binding protein were selected. Nitric oxide reductase has been linked to pathogenesis in other microorganisms [40] and was 2-fold down regulated, along with $c d \operatorname{PadR} 1$ when compared to the historical $C$. difficile strain 630 [41]. Another representative promoter for EMSA study from within $c d$ PadR1 genome neighborhood is upstream of a gene encoding a Spo0B-associated GTP-binding protein (obg, CDR20291_1001) whose homologue was 2-fold down regulated following pig loop infection with the historical strain C. difficile 630 [42]. $c d$ PadR1 bound $\mathrm{P}_{n o r V}$ and $\mathrm{P}_{o b g}$ in vitro (Fig. $6 \mathrm{~d}$ and e, respectively). The migration patterns for $\mathrm{P}_{n o r V}$ and $\mathrm{P}_{o b g}$ differ from that of $\mathrm{P}_{\text {cdpadR1 }}$ (Fig. 6c). For all promoters examined, slower migrating complexes appeared at increasing protein concentrations, which suggests that $c d \mathrm{PadR} 1$ binds to multiple sites in the upstream region of the gene. However, the complexes formed when $c d$ PadR1 is incubated with promoters other than its own are smaller and it appears that a level of saturation, wherein only one large complex is formed, is not reached as it is for $\mathrm{P}_{\text {cdpadR1. }}$. It is well understood that transcription regulators bind a relatively limited set of DNA sequences [43], a concept that we explored for $c d$ PadR1 and $\mathrm{P}_{c d P a d R 1}$ (Fig. 4), as well as a predicted binding motif ( $c d$ PadR1 motif, Fig. 5). Both $\mathrm{P}_{\text {norV }}$ and $\mathrm{P}_{\text {obg }}$ have only one half of the inverted repeat within the $c d$ PadR1 motif (Fig. 6a and b). However, it is unclear whether only one half-site is sufficient to initiate binding to these promoters or if perhaps the binding is nonspecific and related to local DNA structure or AT content. Therefore, we examined binding specificity

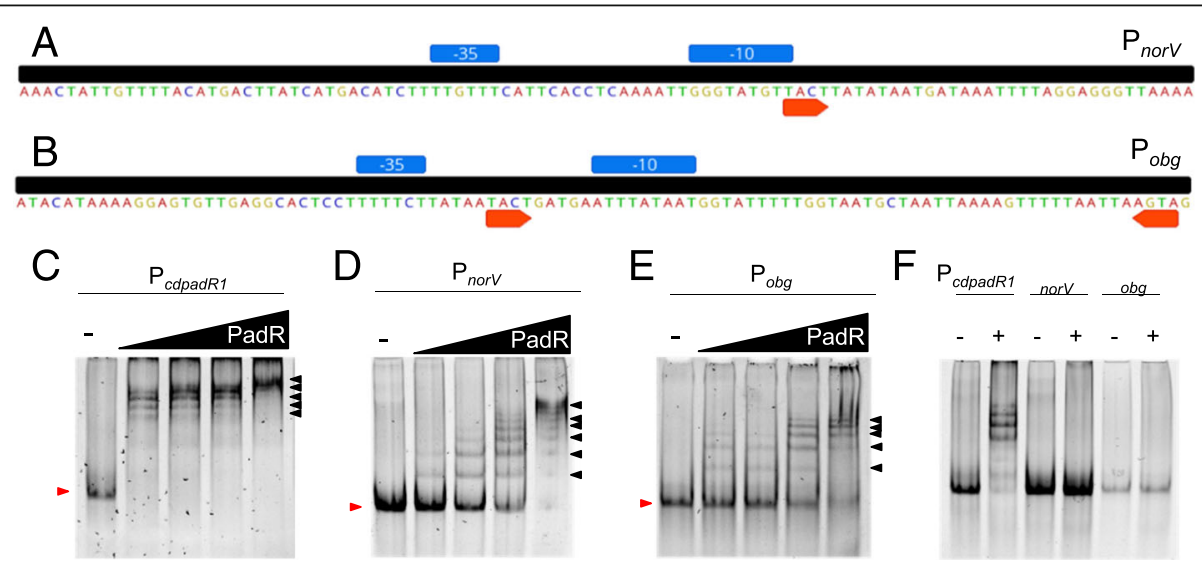

Fig. 6 EMSAs of $c d P a d R 1$ binding the cdpadR1 promoter $\left(P_{c d p a d R 1}\right)$, a norV promoter $\left(P_{\text {nor }}\right)$, and $\mathbf{b}$ obg promoter $\left(P_{\text {obg }}\right)$. The predicted - 10 and -35 sites are indicated in blue boxes above the sequence. Orange arrows indicate the inverted repeats TACT/AGTA. c-e Final $P_{c d p a d R 1}, P_{\text {norv, }}$ and $P_{\text {obg }}$ (100 bp) concentration in the reaction was $0.1 \mu \mathrm{M}$. cdPadR1 was 5, 10, 20, and 40-fold excess over dsDNA. The minus (-) lane contains dsDNA and no cdPadR1. Shifted DNA-protein complexes are annotated with a black arrow and unbound dsDNA migration is marked with a red arrow. $\mathbf{f}$ For this EMSA, the final heparin concentration was 4-fold of the standard EMSA concentration used throughout this research. The + lane contains 40-fold excess cdPadR1 over dsDNA 
using increased amounts of heparin as a competitor for $c d$ PadR1 binding (Fig. 6f). When a 4-fold higher concentration of heparin was present in the binding reaction of cdPadR1 to $\mathrm{P}_{\text {nor }}$ or $\mathrm{P}_{\text {obg }}$ a shifted complex was no longer detected at 40-fold protein over dsDNA (Fig. 6f). Under the same conditions, $c d \operatorname{PadR} 1$ still bound its own promoter, though the larger complexes were no longer detected. That $c d$ PadR1, a small HTH DNA binding protein, would bind other $100 \mathrm{bp}$ predicted promoter regions non-specifically could be explained using the theoretical model termed one-dimensional diffusion, or "sliding". During one-dimensional diffusion, the transcriptional regulator searches for specific binding sites along the DNA remaining in contact with the DNA due to nonspecific interactions [44-46]. It is, therefore, likely that a more specific level of binding requires the full $c d \operatorname{PadR} 1$ motif. So, while $c d$ PadR1 does, in fact, bind $\mathrm{P}_{\text {nor } V}$ and other $100 \mathrm{bp}$ AT-rich promoters in vitro (Additional file 6: Table S1), no conclusions can be made regarding the regulation of this or any other promoters tested based on EMSA alone. Coupled with the recent expression studies, however, in vitro binding assays suggest that further study into the regulation of expression of these genes, especially nor $V$, is warranted.

Role of the conserved $\mathrm{W}$ residue in $c d \mathrm{PadR} 1$ DNA binding It was suggested previously that the conformational changes elicited by drug binding between $\alpha 4 / \alpha 4^{\prime}$ could affect DNA binding and that a conserved tryptophan (W) in $\alpha 4$ was directly involved in drug binding; an indirect role of this W residue was indicated in DNA binding [11]. We examined the effect of this conserved W at residue 94 (W94) in $c d$ PadR1 on DNA binding in vitro (Fig. 7). When W94 is replaced with an alanine $\left(c d \operatorname{PadR} 1^{\mathrm{W} 94 \mathrm{~A}}\right)$, the majority of binding along with the higher order complexes observed for $c d \mathrm{PadR} 1^{\mathrm{WT}}$ binding to $\mathrm{P}_{c d p a d R 1}$ are lost (Fig. 7). Dimerization was not effected as detected by size exclusion chromatography coupled with multi-angle light scattering detection (SEC-MALS, Additional file 1:

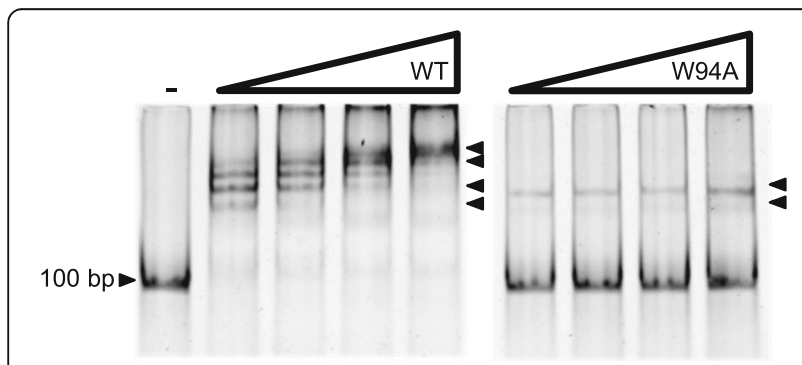

Fig. 7 EMSA of $c d P a d R 1^{W T}$ and $c d P a d R 1^{W 94 A}$ binding $P_{\text {cdpadR1. }}$. Protein-free controls are indicated with a minus sign (-). $100 \mathrm{bp}$ $P_{\text {cdpadR } 1}$ DNA $(0.05 \mu \mathrm{M})$ was used in EMSA to determine binding of $c d P a d R 1$ to its own promoter at increasing concentrations $(0.25,0.50$, 1.0, and $2.0 \mu \mathrm{M})$ of protein
Figure S1). These results suggest that, while the conserved W does not affect dimerization, it does inhibit DNA binding in vitro in a way that is not entirely clear while further supporting a role of the conserved W in DNA binding. The suggested mechanism by Madoori et al wherein the DNA binding helices of LmrR putatively rotate away from each other when the effector-binding/oligomerizatoin domain is perturbed at the conserved W residue is further supported as the mechanism of lowered DNA binding affinity by the results presented here.

\section{Conclusion}

We have determined the $1.9 \AA$ resolution crystal structure of $c d P a d R 1$, which revealed that it is in the PadR-s2 subfamily of PadR transcriptional regulators with other structurally and functionally characterized PadR-like regulators from $B$. cereus ( $b c \mathrm{PadR} 1$ and $b c \mathrm{PadR} 2)$ and L. lactis (LmrR). In vitro protein-DNA binding experiments demonstrate that $c d \operatorname{PadR} 1$ binds a region comprised of the inverted repeats TACT/AGTA and an AT-rich core, GTACTAT $\left(\mathrm{N}_{2}\right)$ $\operatorname{ATTATA}(\mathrm{N}) \underline{\mathrm{AGTA}}$, within its own promoter. These predicted binding sites are present in the $c d \operatorname{PadR} 1$ homologue CD630_1154, suggesting that these transcription regulators are functional homologues as well. $c d$ PadR1 appears to be part of a hierarchical gene regulatory network in $C$. difficile. Furthermore, $c d$ PadR1 non-specifically associates with longer DNA fragments that may facilitate promoter and motif searching. Mutation of the highly conserved $\mathrm{W}$ in the $\alpha 4$ helical region, which is predicted to be involved in multidrug recognition and dimerization in LmrR, resulted in alterations of $c d \operatorname{PadR} 1$ binding to the predicted binding motif, potentially due to tighter constraints on spacing of the inverted repeats as well as a loss of higher order oligomerization. Complementary in vivo studies of $c d \operatorname{PadR} 1$ will allow for a better understanding of its regulatory network.

\section{Additional files}

Additional file 1: Figure S1. (A) Chromatogram from size exclusion chromatography (SEC) run performed on a Superdex 200 Increase 10/300 GL column connected to an ÄKTA Pure 25 (GE Healthcare). The black line represents the calibration standard mix with molecular weights of standards labeled. The blue and red lines indicate the elution profile for $c d P a d R 1^{W T}$ and $c d P a d R 1^{\text {W94A }}$, respectively (13.5 kDa monomer size for both). (B) Molar mass versus elution time of $c d P a d R 1^{\text {WT }}$ and $c d P a d R 1^{\text {W94A }}$ from SEC (as described) coupled with multi-angle light scattering (MALS) detection. Red lines indicate MALS signal (LS) and green lines indicate UV detection. $c d P a d R 1^{\text {WT }}$ and $c d P a d R 1^{\text {W94A }}$ both dimers with molecular weights (MW) of approximately 30 and $27 \mathrm{kDa}$, respectively (monomeric cdPadR1 MW is $13.5 \mathrm{kDa}$ ). (PPTX $222 \mathrm{~kb}$ )

Additional file 2: Figure S2. EMSA of $c d P a d R 1$ binding different fragments of $P_{c d p a d R 1}$, that contain the inverted repeats TACT/AGTA with 4 bp overhang on the $5^{\prime}$ and 3 ' end of inverted repeats. Each dsDNA contains a different number of nucleotides between TACT/AGTA (addition of a central alanine). EMSAs were conducted as described for $100 \mathrm{bp}$ and smaller dsDNA fragments in the materials and methods. A 
complete list of nucleotides tested is available in the table below the EMSA gels. Inverted repeats are underlined. For Pr101, the AT rich region that was mutated is indicated in bold. The - lane contains DNA only and the + lane contains 10-fold cdPadR1 in excess over DNA. (PPTX $256 \mathrm{~kb}$ )

Additional file 3: Table S2. List of GLAM2Scan [24] results for scan of the Clostridium difficile R20291 genome for cdPadR1 motif (Fig. 5a). The.xIsx file includes the locus ID for the gene(s) nearest the motif (column A); the gene annotation, if applicable, or the indication that this motif is within an open reading frame (ORF) or between two convergent genes (column B); the genomic placement (start, column $C$ and end, column D); indication of whether the motif is on the leading (+) or lagging (-) strand (column E); the motif sequence generated by GLAM2Scan (column F); and the score generated by GLAM2Scan that indicates how well the sequence fits the cdPadR1 motif (column G). (XLSX $49 \mathrm{~kb}$ )

Additional file 4: Table S3. List of GLAM2Scan [24] results for scan of the Clostridium difficile R20291 genome for cdPadR1 motif (Fig. 5a) for promoters upstream of genes predicted to be involved in transport/efflux and sporulation. The .xlsx file includes the locus ID for the gene(s) nearest the motif (column A); the gene annotation (column B); the genomic placement (start, column C and end, column D); indication of whether the motif is on the leading (+) or lagging (-) strand (column E); the motif sequence generated by GLAM2Scan (column F); and the score generated by GLAM2Scan that indicates how well the sequence fits the $c d P a d R 1$ motif (column G). (XLSX $39 \mathrm{~kb}$ )

Additional file 5: Figure S3. EMSA of $c d P a d R 1$ binding the $21 \mathrm{bp}$ fragment (Pr68, Additional file 4: Table S1) that contains the inverted repeats TACT/AGTA with 11 nucleotides in between from within its own promoter. Protein-free controls are indicated with a minus sign (-). 21 bp $P_{\text {cdpadR } 1}$ DNA $(0.25 \mu \mathrm{M})$ was used in a reaction with increasing concentrations of $c d P a d R 1$ (2.5, 5.0, and $10.0 \mu \mathrm{M})$. (PPTX $3693 \mathrm{~kb})$

Additional file 6: Table S1. Full list of oligonucleotides that were annealed and used in EMSA studies with cdPadR1. The .xlsx file includes the arbitrary number assigned to each oligonucleotide (column A); the genomic placement of the minimum nucleotide (column B); length in base pairs (bp, column C); indication of binding $(+)$ or no binding detected (-) (column D); locus tag associated with the gene downstream of the oligonucleotide (column E): the annotated gene downstream of the oligonucleotide (column F); and the oligonucleotide sequence $5^{\prime}$ to 3' (column G). (XLSX 50 kb)

\section{Abbreviations}

A: Alanine; Å: Ångström; bc: Bacillus cereus; bcPadR1: Bacillus cereus locus ID BC4206; bcPadR2: Bacillus cereus locus ID BCE3449; bp: Base pairs; C: Celsius; CCD: Charged coupled device; $c d$ : Clostridium difficile; $\mathrm{CdP}_{1590}$ : Promoter for locus ID CDR20291_1590; cdP 1882: Promoter for locus ID CDR20291_1882; cdP 2322: Promoter for locus ID CDR20291_2322; cdPadR1: Locus ID CDR20291_0991; ct: Clostridium thermocellum; C-terminal: Carboxy-terminal; DNA: Deoxyribonucleic acid; dsDNA: Double stranded deoxyribonucleic acid; DTT: Dithiothreitol; EMSA: Electrophoretic mobility shift assay;

gDNA: Genomic deoxyribonucleic acid; GLAM2: Gapped local alignment of motifs version 2; GTP: Guanosine triphosphate; H33342: Hoeschst 33342; HEPES: 4-(2-hydroxyethyl)-1-piperazineethanesulfonic acid; HTH: Helix-turnhelix; ID: Identification; I-I: Indole-indole; I-P: Indole-phenol; KCl: Potassium chloride; L: Liter; LmrR: Lactococcal multidrug resistant transcription regulator; M: Molar; MALS: Multi-angle light scattering; MCSG: Midwest Center for Structural Genomics; MEME: Multiple Em for Motif Elicitation; MgCL2: Magnesium chloride; mM: Millimolar; MW: Molecular weight; N: Any nucleotide; NaCl: Sodium chloride; $\mathrm{NaH}_{2} \mathrm{PO}_{4}$ : Sodium phosphate; $\mathrm{NaOH}$ : Sodium hydroxide; norV: Nitric oxide reductase gene; N-terminal: Amino-terminal; obj: Gene encoding SpoOB; ORF: Open reading frame; PadR: Phenolic acid decarboxylase regulator; PadR-s1: Subfamily of the PadR protein family; PadR-s2: Subfamily of the PadR protein family; $P_{\text {cdpadk1: }}$ Promoter of cdpadR1 (locus ID CDR20291_0991);

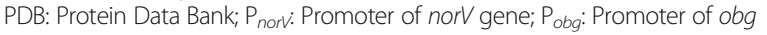
gene; P-P: Phenol-phenol; Pr: Promoter; qRT-PCR: quantitative reverse transcriptase polymerase chain reaction; RMSD: root mean square deviation; SpoOB: sporulation initiation phosphotrasferase B; ssDNA: single stranded deoxyribonucleic acid; TB: tris-borate; W: tryptophan; wHTH: winged helix-turnhelix; WT: wild-type; $\mu \mathrm{L}$ : microliter; $\mu \mathrm{M}$ : micromolar

\section{Acknowledgements}

We would like to thank Ana Gonzalez for help with the data collection during the RapiData 2015 course. We also thank the 2015 RapiData Course for access to the Stanford Synchrotron Radiation Lightsource (SSRL) beamline (BL-14) for diffraction data collection. SSRL is supported by the U.S. Department of Energy, Office of Science, Office of Basic Energy Sciences under Contract No. DE-AC02-76SF00515. The SSRL Structural Molecular Biology Program is supported by the DOE Office of Biological and Environmental Research, and by the National Institutes of Health, National Institute of General Medical Sciences (including P41GM103393). Additionally, we would also like to thank Dr. Jimmy Ballard for Clostridium difficile gDNA. We are also grateful to Neda Hessami (OU Michael F. Price Institute for Structural Biology) for large-scale sample preparations and helpful discussions. We thank Fares Najar, Jamie Sykes, Samantha Powell, Bing Wang and Skyler Hebdon (University of Oklahoma); and Steve Almo, James Love, and Vern Schramm (Albert Einstein College of Medicine) for thoughtful discussions. We are grateful to Dr. Eliza Ruben for sample processing assistance in the OU Protein Production Core facility, which is funded by an Institutional Development Award (IDeA) from the National Institute of General Medical Sciences of the National Institutes of Health under grant number P20GM103640 to Ann H. West. Initial crystallization trails were carried out in the OU Macromolecular Crystallography Laboratory which is partially funded by National Science Foundation Major Research Instrumentation award 092269.

\section{Funding}

This study was supported by private funding from The Price Family Foundation (New York, NY). The funding organization had no involvement in study design, data analysis or interpretation.

\section{Availability of data and materials}

All expression plasmids utilized in this study are available upon request. Primer/Oligonucleotide sequences are available in Additional file 4: Table S1. Atomic coordinates were submitted to the Protein Data Bank under accession number 5DYM (http://www.rcsb.org/pdb/home/home.do) and will be publically available upon publication. All additional data generated or analyzed during this study are included in this published article [and its supplementary information files].

\section{Authors' contributions}

$\mathrm{Cl}$ is responsible for all protein purification and EMSA experiments as well as X-ray data collection. SM and LT assisted $\mathrm{Cl}$ in structure determination. AW, GR and EA are the project Pl's that were involved in study design and oversight. All authors participated in manuscript preparation. All authors read and approved the final manuscript.

\section{Competing interests}

The authors declare that they have no competing interests.

\section{Consent for publication}

Not applicable.

\section{Ethics approval and consent to participate} Not Applicable.

\section{Author details}

'Price Family Foundation Institute of Structural Biology and Department of Microbiology and Plant Biology, University of Oklahoma, 770 Van Vleet Oval, Norman, OK 73019, USA. ${ }^{2}$ Price Family Foundation Institute of Structural Biology and Department of Chemistry and Biochemistry, University of Oklahoma, 101 Stephenson Parkway, Norman, OK 73019, USA.

\section{Received: 4 August 2016 Accepted: 24 September 2016}

\section{Published online: 04 October 2016}

\section{References}

1. Tickler IA, Goering RV, Whitmore JD, Lynn AN, Persing DH, Tenover FC, Healthcare Associated Infection C. Strain types and antimicrobial resistance patterns of Clostridium difficile isolates from the United States, 2011 to 2013. Antimicrob Agents Chemother. 2014;58(7):4214-8.

2. Rupnik M, Wilcox MH, Gerding DN. Clostridium difficile infection: new developments in epidemiology and pathogenesis. Nat Rev Microbiol. 2009;7(7):526-36. 
3. He M, Sebaihia M, Lawley TD, Stabler RA, Dawson LF, Martin MJ, Holt KE, Seth-Smith HM, Quail MA, Rance R, et al. Evolutionary dynamics of Clostridium difficile over short and long time scales. Proc Natl Acad Sci U S A. 2010;107(16):7527-32.

4. Lawley TD, Croucher NJ, Yu L, Clare S, Sebaihia M, Goulding D, Pickard DJ, Parkhill J, Choudhary J, Dougan G. Proteomic and genomic characterization of highly infectious Clostridium difficile 630 spores. J Bacteriol. 2009;191(17):5377-86.

5. Barthelmebs L, Lecomte B, Divies C, Cavin JF. Inducible metabolism of phenolic acids in Pediococcus pentasaceus is encoded by an autoregulated operon which involves a new class of negative transcription regulator. J Bacteriol. 2000;182(23):8.

6. Tran NP, Gury J, Dartois V, Nguyen TK, Seraut H, Barthelmebs L, Gervais P, Cavin JF. Phenolic acid-mediated regulation of the padC gene, encoding the phenolic acid decarboxylase of Bacillus subtilis. J Bacteriol. 2008;190(9):3213-24.

7. Nguyen TK, Tran NP, Cavin JF. Genetic and biochemical analysis of PadR-padC promoter interactions during the phenolic acid stress response in Bacillus subtilis 168. J Bacteriol. 2011;193(16):4180-91.

8. De Silva RS, Kovacikova G, Lin W, Taylor RK, Skorupski K, Kull FJ. Crystal structure of the virulence gene activator AphA from Vibrio cholerae reveals it is a novel member of the winged helix transcription factor superfamily. J Biol Chem. 2005;280(14):13779-83.

9. Agustiandari H, Lubelski J, van den Berg van Saparoea HB, Kuipers OP, Driessen AJ. LmrR is a transcriptional repressor of expression of the multidrug $A B C$ transporter LmrCD in Lactococcus lactis. J Bacteriol. 2008;190(2):759-63.

10. Fibriansah G, Kovacs AT, Pool TJ, Boonstra M, Kuipers OP, Thunnissen AM. Crystal structures of two transcriptional regulators from Bacillus cereus define the conserved structural features of a PadR subfamily. PLoS One. 2012;7(11):e48015

11. Madoori PK, Agustiandari H, Driessen AJ, Thunnissen AM. Structure of the transcriptional regulator $\mathrm{LmrR}$ and its mechanism of multidrug recognition. EMBO J. 2009;28:11.

12. Grande Burgos MJ, Kovacs AT, Mironczuk AM, Abriouel H, Galvez A, Kuipers OP. Response of Bacillus cereus ATCC 14579 to challenges with sublethal concentrations of enterocin AS-48. BMC Microbiol. 2009;9:227.

13. Takeuchi K, Tokunaga Y, Imai M, Takahashi H, Shimada I. Dynamic multidrug recognition by multidrug transcriptional repressor $\mathrm{LmrR}$. Sci Rep. 2014;4:6922.

14. Dembek M, Stabler RA, Witney AA, Wren BW, Fairweather NF. Transcriptional analysis of temporal gene expression in germinating Clostridium difficile 630 endospores. PLoS One. 2013;8(5):e64011.

15. Emerson JE, Stabler RA, Wren BW, Fairweather NF. Microarray analysis of the transcriptional responses of Clostridium difficile to environmental and antibiotic stress. J Med Microbiol. 2008;57(Pt 6):757-64.

16. Karr EA. The methanogen-specific transcription factor MsvR regulates the fpaA-rlp-rub oxidative stress operon adjacent to msvR in Methanothermobacter thermautotrophicus. J Bacteriol. 2010;192(22):5914-22.

17. Kabsch W. Xds. Acta Crystallogr D Struct Biol. 2010;66(Pt 2):125-32.

18. Winn MD, Ballard CC, Cowtan KD, Dodson EJ, Emsley P, Evans PR, Keegan RM, Krissinel EB, Leslie AG, McCoy A, et al. Overview of the CCP4 suite and current developments. Acta Crystallogr D Struct Biol. 2011;67(Pt 4):235-42.

19. McCoy AJ, Grosse-Kunstleve RW, Adams PD, Winn MD, Storoni LC, Read RJ. Phaser crystallographic software. J Appl Crystallogr. 2007;40(Pt 4):658-74.

20. Adams PD, Afonine PV, Bunkoczi G, Chen VB, Davis IW, Echols N, Headd JJ, Hung LW, Kapral GJ, Grosse-Kunstleve RW, et al. PHENIX: a comprehensive Python-based system for macromolecular structure solution. Acta Crystallogr D Biol Crystallogr. 2010;66(Pt 2):213-21.

21. Emsley P, Lohkamp B, Scott WG, Cowtan K. Features and development of Coot. Acta Crystallogr D Biol Crystallogr. 2010;66(Pt 4):486-501.

22. Schrödinger LLC. The PyMOL molecular graphics system, version 1.3 r1.2010. 2010.

23. Ho SN, Hunt HD, Horton RM, Pullen JK, Pease LR. Site-directed mutagenesis by overlap extension using the polymerase chain reaction. Gene. 1989;77(1):51-9.

24. Frith MC, Saunders NF, Kobe B, Bailey TL. Discovering sequence motifs with arbitrary insertions and deletions. PLoS Comput Biol. 2008;4(4):e1000071.

25. Kearse M, Moir R, Wilson A, Stones-Havas S, Cheung M, Sturrock S, Buxton S, Cooper A, Markowitz S, Duran C, et al. Geneious Basic: an integrated and extendable desktop software platform for the organization and analysis of sequence data. Bioinformatics. 2012;28(12):1647-9.
26. Kelley LA, Sternberg MJ. Protein structure prediction on the Web: a case study using the Phyre server. Nat Protoc. 2009:4(3):363-71.

27. Holm L, Rosenström P. Dali server: conservation mapping in 3D. Nucleic Acids Res. 2010;38(Web Server issue):W545-549.

28. Aravind L, Anantharaman V, Balaji S, Babu MM, lyer LM. The many faces of the helix-turn-helix domain: transcription regulation and beyond. FEMS Microbiol Rev. 2005;29(2):231-62.

29. Krissinel E, Henrick K. Inference of macromolecular assemblies from crystalline state. J Mol Biol. 2007;372(3):774-97.

30. Pettersen EF, Goddard TD, Huang CC, Couch GS, Greenblatt DM, Meng EC, Ferrin TE. UCSF Chimera-a visualization system for exploratory research and analysis. J Comput Chem. 2004;25(13):1605-12.

31. Gury J, Barthelmebs L, Tran NP, Divies C, Cavin JF. Cloning, deletion, and characterization of PadR, the transcriptional repressor of the phenolic acid decarboxylase-encoding padA gene of Lactobacillus plantarum. J App Environ Microbiol. 2004;70(4):2146-53.

32. Pareja E, Pareja-Tobes P, Manrique M, Pareja-Tobes E, Bonal J, Tobes R. Extra Train: a database of extragenic regions and transcriptional information in prokaryotic organisms. BMC Microbiol. 2006;6:10.

33. van Hijum SA, Medema MH, Kuipers OP. Mechanisms and evolution of control logic in prokaryotic transcriptional regulation. Microbiol Mol Biol Rev. 2009;73(3):481-509. Table of Contents.

34. Brennan R, Matthews BW. The helix-turn-helix DNA binding motif. J Biol Chem. 1989;264(4):4

35. Fried MG. Measurement of protein-DNA interaction parameters by electrophoresis mobility shift assay. Electrophoresis. 1998;10:366-76.

36. Seshasayee ASN, Bertone P, Fraser GM, Luscombe NM. Transcriptional regulatory networks in bacteria: from input signals to output responses. Curr Opin Microbiol. 2006;9(5):511-9.

37. Lozada-Chávez I, Janga SC, Collado-Vides J. Bacterial regulatory networks are extremely flexible in evolution. Nucleic Acids Res. 2006;34(12):3434-45.

38. Solovyev V, Salamov A. Automatic annotation of microbial genomes and metagenomic sequences. In: Metagenomics and its Applications in Agriculture, Biomedicine and Environmental Studies. New York: Edited by Li, RW Nova Science Publishers; 2011: 61-78.

39. Gralla JD, Collado-Vides J. Organization and function of transcription regulatory elements. In: Niedhardt FC, Curtiss R, Ingraham III JL, Lin ECC, Low KB, Magasanik B, editors. Escherichia coli and Salmonella: Cellular and Molecular Biology. Washington, DC: ASM Press; 1996. p. 1232-45.

40. Shimizu T, Tsutsuki H, Matsumoto A, Nakaya H, Noda M. The nitric oxide reductase of enterohaemorrhagic Escherichia coli plays an important role for the survival within macrophages. Mol Microbiol. 2012;85(3):492-512.

41. Scaria J, Mao C, Chen JW, McDonough SP, Sobral B, Chang YF. Differential stress transcriptome landscape of historic and recently emerged hypervirulent strains of Clostridium difficile strains determined using RNA-seq. PLoS One. 2013:8(11):e78489.

42. Scaria J, Janvilisri T, Fubini S, Gleed RD, McDonough SP, Chang YF. Clostridium difficile transcriptome analysis using pig ligated loop model reveals modulation of pathways not modulated in vitro. J Infect Dis. 2011;203(11):1613-20.

43. Berg OG, von Hippel PH. Selection of DNA binding sites by regulatory proteins. Trends Biochem Sci. 1988;13(6):207-11.

44. Sela I, Lukatsky DB. DNA sequence correlations shape nonspecific transcription factor-DNA binding affinity. Biophys J. 2011;101(1):160-6.

45. Cherstvy AG, Kolomeisky AB, Kornyshev AA. Protein-DNA interactions: reaching and recognizing the targets. J Phys Chem B. 2008;112:4741-50.

46. Hu T, Grosberg AY, Shklovskii BI. How proteins search for their specific sites on DNA: the role of DNA conformation. Biophys J. 2006;90(8):2731-44. 